\title{
ANTI-SYNCHRONIZATON OF TWO DIFFERENT HYPERCHAOTIC SYSTEMS VIA ACTIVE GENERALIZED BACKSTEPPING METHOD
}

\author{
Ali Reza Sahab ${ }^{1}$ and Masoud Taleb Ziabari ${ }^{2}$ \\ ${ }^{1}$ Faculty of Engineering, Electrical Engineering Group, Islamic Azad University, Lahijan \\ Branch, Iran. \\ sahabeliau.ac.ir \\ ${ }^{2}$ Faculty of Engineering, Computer Engineering Group, Ahrar University, Rasht, Iran. \\ m.t.ziabari@gmail.com
}

\begin{abstract}
This paper presents hyperchaos anti-synchronization of different hyperchaotic systems using Active Generalized Backstepping Method (AGBM). The proposed technique is applied to achieve hyperchaos antisynchronization for the Lorenz and Lu dynamical systems. Generalized Backstepping Method (GBM) is similarity to Backstepping and more applications in systems than it. Bckstepping method is used only to strictly feedback systems but GBM expand this class. The hybrid active control method and generalized backstepping method forces the system error to decay to zero rapidly that it causes the system to have a short setteling time, overshoot.
\end{abstract}

\section{KEYWORDS}

Active Generalized Backstepping Method, Anti-Synchronization, Hyperchaotic Lorenz System, Hyperchaotic Lu System, Lyapunov Direct Method.

\section{INTRODUCTION}

An interesting phenomenon of nonlinear systems is chaos. In recent years, studies of chaos and hyperchaos generation, control and synchronization have attracted considerable attentions, laser, nonlinear circuit and neural network, etc [1-13]. Therefore, various effective methods have been proposed one the past decades to achieve the control and stabilization of chaotic system, such as Robust Control [1], the sliding method control [2], linear and nonlinear feedback control [3], adaptive control [4], active control [5], backstepping control [6] and generalized backsteppig method control [7-9], ect. Hyperchaotic system has more complex dynamical behaviors than chaotic system. Historically, the more well know hyperchaotic systems are the 4D hyperchaotic Rossler system [14], the 4D hyperchaotic Chua's circuit [15], the generalized Lorenz system [16], Chen system [17] and Lu system[18]. Synchronization of chaotic systems has become more and more interesting topics to engineering and science communities [19-27]. The concept of synchronization has been extended to the scope, such as phase synchronization [28], lag synchronization [29] and even anti-synchronization (anti-phase synchronization) [30-32].

This paper is organized as follows: in Section 2, studies the Generalized Backstepping Method. In Section 3, involves the basic properties for the Hyperchaotic systems. In Section 4, studies the anisynchronization of the two diferent hyperchaotic system with an Active Generalized Backstepping Method. In Section 5, numerical simulation of output presented. Conclusion are given in final section. 


\section{The Generalized BACKSTEPPING METHOD}

Generalized Backstepping Method [7-9] will be applied to a certain class of autonomous nonlinear systems which are expressed as follow

$\left\{\begin{array}{l}\dot{X}=F(X)+G(X) \eta \\ \dot{\eta}=f_{0}(X, \eta)+g_{0}(X, \eta) u\end{array}\right.$

In which $\eta \epsilon \Re$ and $x=\left[x_{1}, x_{2}, \cdots, x_{n}\right] \epsilon \Re$. In order to obtain an approach to control these systems, we may need to prove a new theorem as follow.

Theorem : suppose equation (1) is available, then suppose the scalar function $\varphi_{i}(x)$ for the $i_{t h}$ state could be determined i a manner which by inserting the $i_{t h}$ term for $\eta$, the function $v(x)$ would be a positive definite equation (3) with negative definite derivative.

$V(X)=\frac{1}{2} \sum_{i=1}^{n} x_{i}^{2}$

Therefore, the control signal and also the general control lyapunov function of this system can be obtained by equation (3),(4).

$$
\begin{aligned}
& u=\frac{1}{g_{0}(X, \eta)}\left\{\begin{array}{c}
\sum_{i=1}^{n} \sum_{j=1}^{n} \frac{\partial \varphi_{i}}{\partial x_{j}}\left[f_{i}(X)+g_{i}(X) \eta\right] \\
-\sum_{i=1}^{n} x_{i} g_{i}(X)-\sum_{i=1}^{n} k_{i}\left[\eta-\varphi_{i}(X)\right]-f_{0}(X, \eta)
\end{array}\right\}, k_{i}>0, i=1,2, \cdots, n \\
& V_{t}(X, \eta)=\frac{1}{2} \sum_{i=1}^{n} x_{i}^{2}+\frac{1}{2} \sum_{i=1}^{n}\left[\eta-\varphi_{i}(X)\right]^{2}
\end{aligned}
$$

\section{SYSTEM DESCRIPTION}

The hyperchaotic Lorenz system [16] is described by

$$
\begin{aligned}
& \dot{x}=a(y-x)+w \\
& \dot{y}=-x z+c x-y \\
& \dot{z}=x y-b z \\
& \dot{w}=-x z+d w
\end{aligned}
$$

Where $a, b, c$ and $d$ are constants. When parameters $a=10, b=\frac{8}{3}, c=28$ and $d=1.3$, the system (5) shows hyperchaotic behavior. See figure 1. 

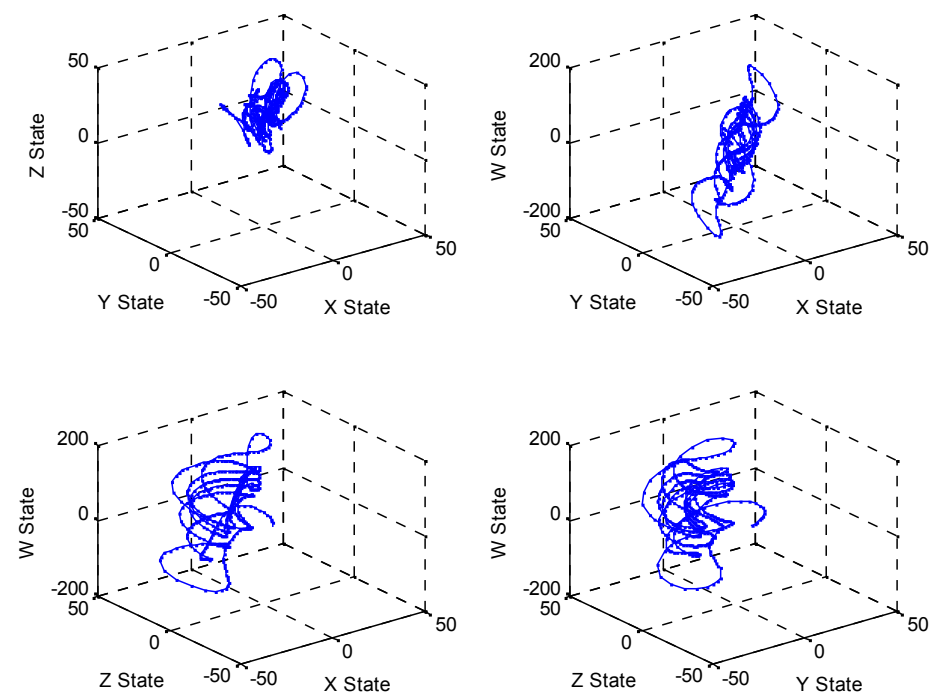

Figure 1. Phase Plane of hyperchaotic Lorenz system.

The hyperchaotic Lu system [18] is described by

$\dot{x}=a(y-x)+w$

$\dot{y}=-x z+c y$

$\dot{z}=x y-b z$

$\dot{w}=x z+d w$

Where $x, y, z$ and $w$ are state variables and $a, b, c$ and $d$ are real constants. When $a=36, b=$ $3, c=20,-0.35<d \leq 1.3$, system (6) has hyperchaotic attractor. See fig 2 .
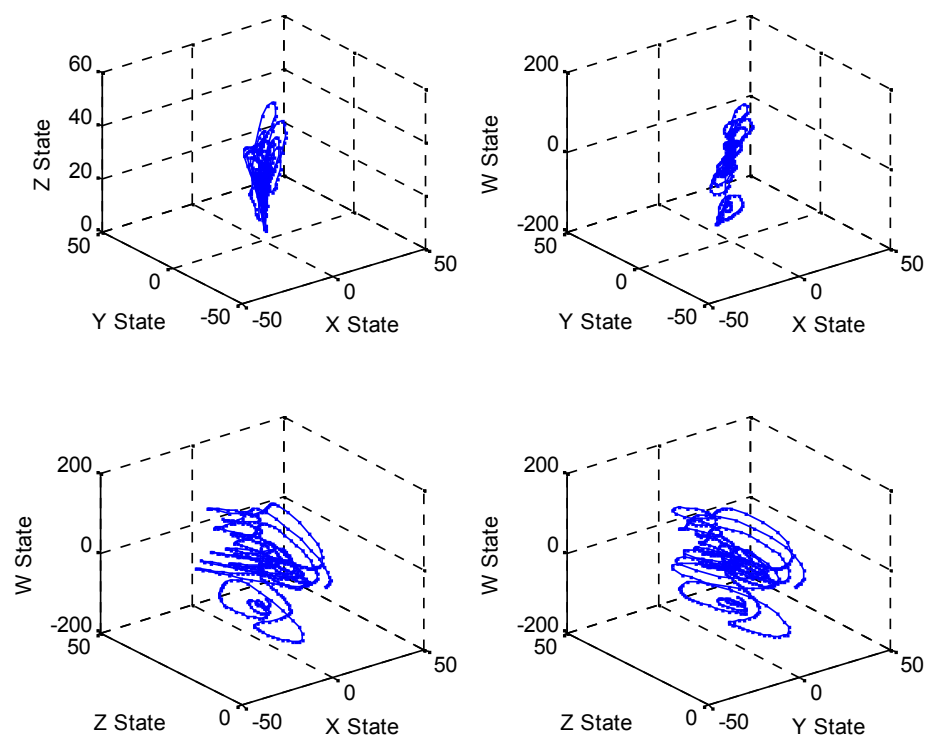

Figure 2. Phase Plane of hyperchaotic Lu system. 


\section{AnTi-SynChronization OF TWO DIFFERENT HYPERCHAOTIC SySTEMS}

In this section, the hybrid active control method and generalized backstepping method is applied to anti-synchronize between the hyperchaotic lorenz system and the hyperchaotic lu system.

Suppose the drive system takes the following from

$$
\begin{aligned}
& \dot{x}_{1}=a_{1}\left(y_{1}-x_{1}\right)+w_{1} \\
& \dot{y}_{1}=-x_{1} z_{1}+c_{1} x_{1}-y_{1} \\
& \dot{z}_{1}=x_{1} y_{1}-b_{1} z_{1} \\
& \dot{w}_{1}=-x_{1} z_{1}+d_{1} w_{1}
\end{aligned}
$$

And the response system is given as follows

$$
\begin{aligned}
& \dot{x}_{2}=a_{2}\left(y_{2}-x_{2}\right)+w_{2}+u_{1}(t) \\
& \dot{y}_{2}=-x_{2} z_{2}+c_{2} y_{2}+u_{2}(t) \\
& \dot{z}_{2}=x_{2} y_{2}-b_{2} z_{2}+u_{3}(t) \\
& \dot{w}_{2}=x_{2} z_{2}+d_{2} w_{2}+u_{4}(t)
\end{aligned}
$$

Where $u_{1}(t), u_{2}(t), u_{3}(t)$ and $u_{4}(t)$ are control functions to be determined for achieving antisynchronization between the two systems (7) and (8).

Define state errors between system (7) and (8) as follows

$$
\begin{aligned}
& e_{x}=x_{1}+x_{2} \\
& e_{y}=y_{1}+y_{2} \\
& e_{z}=z_{1}+z_{2} \\
& e_{w}=w_{1}+w_{2}
\end{aligned}
$$

We obtain the following error dynamical system by adding the drive system (7) with the response system (8).

$$
\begin{aligned}
& \dot{e}_{x}=a_{2}\left(e_{y}-e_{x}\right)+e_{w}+\left(a_{1}-a_{2}\right)\left(y_{1}-x_{1}\right)+u_{1}(t) \\
& \dot{e}_{y}=c_{2} e_{y}-\left(1+c_{2}\right) y_{1}-c_{1} x_{1}-x_{1} z_{1}-x_{2} z_{2}+u_{2}(t) \\
& \dot{e}_{z}=-b_{2} e_{z}+\left(b_{2}-b_{1}\right) z_{1}+x_{1} y_{1}+x_{2} y_{2}+u_{3}(t) \\
& \dot{e}_{w}=d_{2} e_{w}+\left(d_{1}-d_{2}\right) w_{1}+x_{2} z_{2}-x_{1} z_{1}+u_{4}(t)
\end{aligned}
$$

Define the following active control functions $u_{1}(t), u_{2}(t), u_{3}(t)$ and $u_{4}(t)$.

$$
\begin{aligned}
& u_{1}(t)=-\left(a_{1}-a_{2}\right)\left(y_{1}-x_{1}\right)+v_{1}(t) \\
& u_{2}(t)=\left(1+c_{2}\right) y_{1}-c_{1} x_{1}+x_{1} z_{1}+x_{2} z_{2}+v_{2}(t) \\
& u_{3}(t)=-\left(b_{2}-b_{1}\right) z_{1}-x_{1} y_{1}-x_{2} y_{2}+v_{3}(t) \\
& u_{4}(t)=-\left(d_{1}-d_{2}\right) w_{1}+x_{1} z_{1}-x_{2} z_{2}+v_{4}(t)
\end{aligned}
$$

Where $v_{1}(t), v_{2}(t), v_{3}(t)$ and $v_{4}(t)$ are control inputs.

Substituting equation (11) into equation (10) yields.

$$
\begin{aligned}
& \dot{e}_{x}=a_{2}\left(e_{y}-e_{x}\right)+e_{w}+v_{1}(t) \\
& \dot{e}_{y}=c_{2} e_{y}+v_{2}(t) \\
& \dot{e}_{z}=-b_{2} e_{z}+v_{3}(t) \\
& \dot{e}_{w}=d_{2} e_{w}+v_{4}(t)
\end{aligned}
$$


Thus, the error system (12) to be controlled with control inputs $v_{1}(t), v_{2}(t), v_{3}(t)$ and $v_{4}(t)$ as functions of error states $e_{x}, e_{y}, e_{z}$ and $e_{w}$. When system (12) is stabilized by control inputs $v_{1}(t), v_{2}(t), v_{3}(t)$ and $v_{4}(t), e_{x}, e_{y}, e_{z}$ and $e_{w}$ will converage to zeroes as time $t$ tends to infinity. Which implies that system (5) and (6) are anti-synchronized.

To achieve this purpose, we choose control inputs by using generalized backstepping method such that

$$
\begin{aligned}
& v_{1}(t)=0 \\
& v_{2}(t)=-a_{2} e_{x}-\left(k_{1}+c_{2}\right) e_{y} \\
& v_{3}(t)=-k_{2} e_{z} \\
& v_{4}(t)=-e_{x}-\left(k_{3}+d_{2}\right) e_{w}
\end{aligned}
$$

Now, using the gradient optimization of neural network coefficients controllers suitable for use relationship (13) let's examine. In this case, the coefficients $k_{i} ; i=1,2,3$ benefit obtained from the following relationship will come.

$k_{1}(t+1)=\left|k_{1}(t)-\alpha e_{y}\right|$

$k_{2}(t+1)=\left|k_{2}(t)-\alpha e_{z}\right|$

$k_{3}(t+1)=\left|k_{3}(t)-\alpha e_{w}\right|$

Where $\alpha$ the learning rate is would be equal to 0.01 . Initial value of $k_{i} ; i=1,2,3$ are equal to 50 .

\section{NUMERICAL Simulation}

This section presents numerical simulations anti-synchronization of hyperchaotic lorenz system and hyperchaotic lu system. The Active Generalized Backstepping Method (AGBM) is used as an approach to anti-synchronize hyperchaotic lorenz and lu system, eventually the result of this method would be compared with the anti-synchronization result of Nonlinear Control Method (NCM) [32]. We select the parameters of the hyperchaotic lorenz system as $a=10, b=\frac{8}{3}, c=$ $28, d=1.3$ and for the hyperchaotic lu as $a=36, b=3, c=20, d=1.3$, so that these systems exhibits a hyperchaotic behavior. The initial values of the the drive and response systems are $x_{1}(0)=5, y_{1}(0)=8, z_{1}(0)=-1, w_{1}(0)=-3$ and $x_{2}(0)=3, y_{2}(0)=4, z_{2}(0)=$ $5, w_{2}(0)=5$ respectively. The time response of $x, y, z, w$ states for drive system (hyperchaotic Lorenz) and the response system (hyperchaotic $\mathrm{Lu}$ ) via active generalized backstepping method shown in order figure 3 until figure 6. Anti-Synchronization errors $\left(e_{x}, e_{y}, e_{z}, e_{w}\right)$ in hyperchaotic lorenz system and hyperchaotic lu system shown in order figure 7 until figure 10.

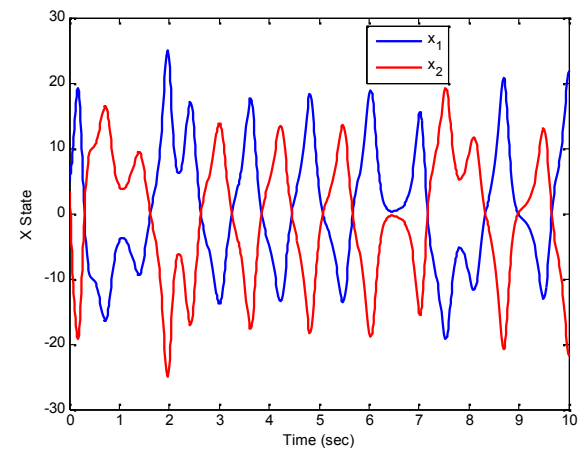

Figure 3. The time response of signals $x_{1}$ and $x_{2}$ for hyperchaotic Lorenz and Lu systems. 
International Journal of Chaos, Control, Modelling and Simulation (IJCCMS) Vol.2, No.1, March 2013

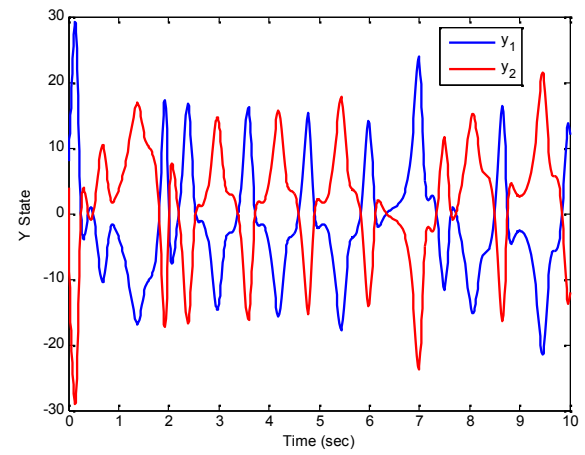

Figure 4. The time response of signals $y_{1}$ and $y_{2}$ for hyperchaotic Lorenz and Lu systems.

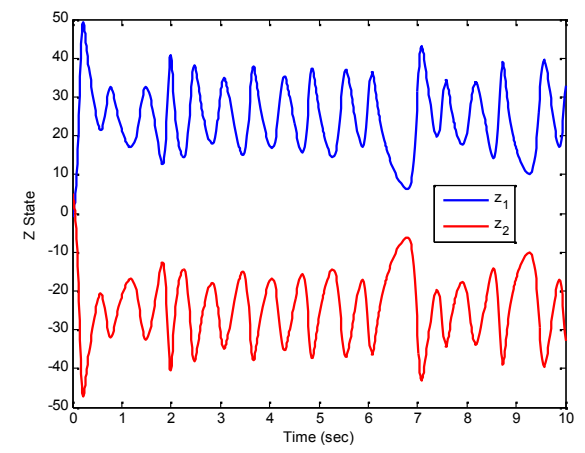

Figure 5. The time response of signals $z_{1}$ and $z_{2}$ for hyperchaotic Lorenz and Lu systems.

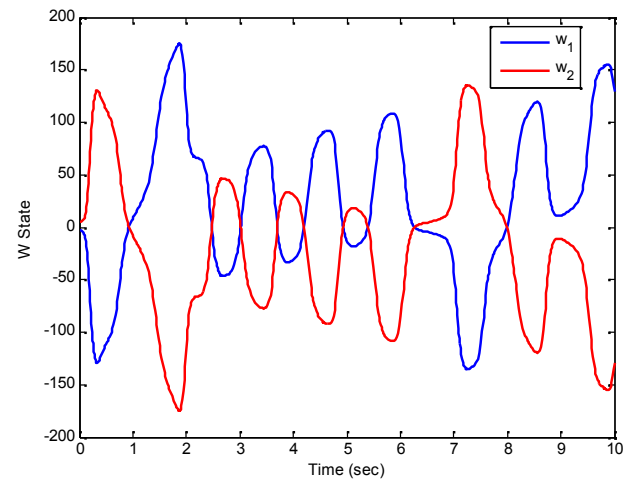

Figure 6. The time response of signals $w_{1}$ and $w_{2}$ for hyperchaotic Lorenz and Lu systems.

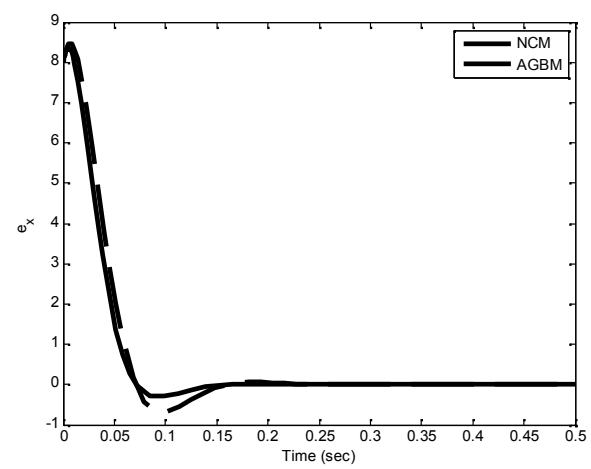

Figure 7. Anti-Synchronization $e_{x}$ in hyperchaotic Lorenz and Lu systems. 


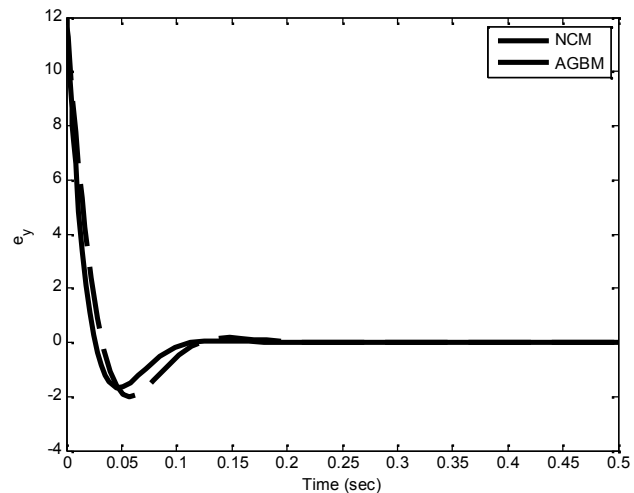

Figure 8. Anti-Synchronization $e_{y}$ in hyperchaotic Lorenz and Lu systems.

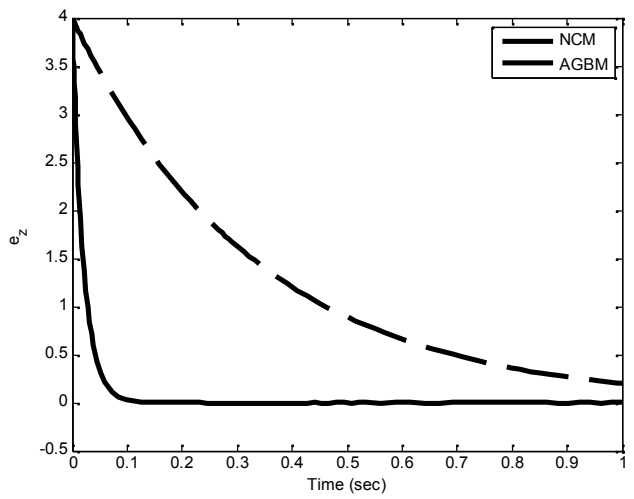

Figure 9. Anti-Synchronization $e_{z}$ in hyperchaotic Lorenz and Lu systems.

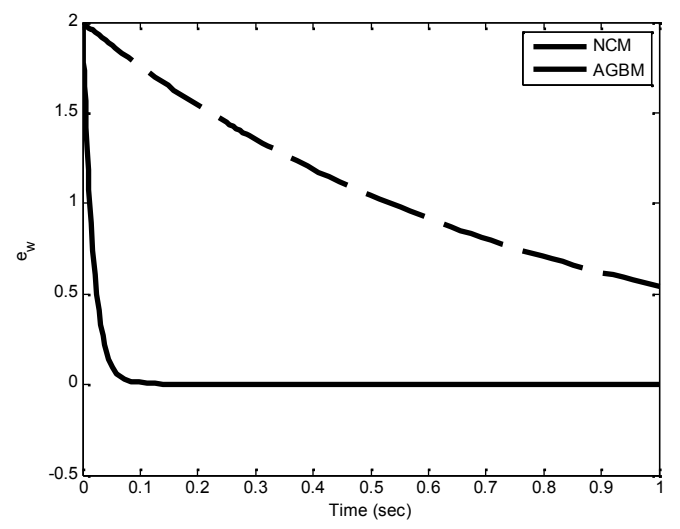

Figure 10. Anti-Synchronization $e_{w}$ in hyperchaotic Lorenz and Lu systems.

\section{CONCLUSIONS}

This study demonstrated that anti-synchronization can coexist in two different hyperchaotic systems ratchets moving in different asymmetric potentials by active generalized backstepping method. Hyperchaotic Lu system is controlled to be anti-synchronized with hyperchaotic Lorenz system. In the Active Generalized Backstepping Method in relation to the Nonlinear Control Method [32], control will be accomplished in a much shorter time and overshoot. The simulations confirm that Anti-Synchronization of two systems operates satisfactorily in presence of the proposed control method. 
International Journal of Chaos, Control, Modelling and Simulation (IJCCMS) Vol.2, No.1, March 2013

\section{REFERENCES}

[1] Chao-Chung Peng, Chieh-Li Chen. Robust chaotic control of Lorenz system by backstepping design. Chaos, Solitons and Fractals 37 (2008) 598-608.

[2] Cheng-Chi Wang, Neng-Sheng Pai, Her-Terng Yau. Chaos control in AFM system using sliding mode control by backstepping design. Commun Nonlinear Sci Numer Simulat 15 (2010) 741-751.

[3] Faqiang Wang, Chongxin Liu. A new criterion for chaos and hyperchaos synchronization using linear feedback control. Physics Letters A 360 (2006) 274-278.

[4] Yongguang Yu, Suochun Zhang. Adaptive backstepping synchronization of uncertain chaotic system. Chaos, Solitons and Fractals 21 (2004) 643-649.

[5] Sinha SC, Henrichs JT, Ravindra BA. A general approach in the design of active controllers for nonlinear systems exhibiting chaos. Int J Bifurcat Chaos 2000;10(1):165-78.

[6] M.T. Yassen. Chaos control of chaotic dynamical systems using backstepping design. Chaos, Solitons and Fractals 27 (2006) 537-548.

[7] Ali Reza Sahab and Mohammad Haddad Zarif. Improve Backstepping Method to GBM. World Applied Sciences Journal 6 (10): 1399-1403, 2009, ISSN 1818-4952.

[8] Sahab, A.R. and M. Haddad Zarif. Chaos Control in Nonlinear Systems Using the Generalized Backstopping Method. American J. of Engineering and Applied Sciences 1 (4): 378-383, 2008, ISSN 1941-7020.

[9] Ali Reza Sahab, Masoud Taleb Ziabari, Seyed Amin Sadjadi Alamdari. Chaos Control via Optimal Generalized Backstepping Method. International Review of Electrical Engineering (I.R.E.E), Vol.5, n.5.

[10] Yu Yongguang, Zhang Suochun. Controlling uncertain Lu system using backstepping design. Chaos, Solitons and Fractals 15 (2003) 897-902.

[11] Ya-Fu Peng, Chun-Fei Hsu. Identification-based chaos control via backstepping design using selforganizing fuzzy neural networks. Chaos, Solitons and Fractals 41 (2009) 1377-1389.

[12] Tzuyin Wu, Min-Shin Chen. Chaos control of the modified Chua's circuit system. Physica D 164 (2002) 53-58.

[13] Prodip Roy, Banshidhar Sahoo and Swarup Poria, SEASONAL VARIATION OF CARRYING CAPACITY ON DISEASED MODEL CAN CAUSE SPECIES EXTINCTION, International Journal of Chaos, Control, Modelling and Simulation (IJCCMS) Vol.1, No.2, December 2012.

[14] O.E. R ssler, An equation for hyperchaos, Phys. Lett. A 71 (1979) 155-157.

[15] T. Kapitaniak, L.O. Chua, Hyperchaotic attractors of unidirectionally coupled Chua's CircuitsInt, J. Bifur. Chaos 4 (1994) 477-482.

[16] Q. Jia, Hyperchaos generated from the Lorenz chaotic system and its control, Phys. Lett. A 366 (2007) 217-222.

[17] Y. Li, G. Chen, W.K.S. Tang, Hyperchaotic Chen's system and its generation, IEEE Trans. Circuits Syst. II 52 (2005) 204-207.

[18] A. Chen, J. Lu, J. Lü, S. Yu, Generating hyperchaotic Lü attractor via state feedback control, Phys. A 364 (2006) 103-110.

[19] U.E. Vincent, A. Ucar, J.A. Laoye, S.O. Kareem. Control and synchronization of chaos in RCLshunted Josephson junction using backstepping design. Physica C 468 (2008) 374-382.

[20] Qiang Jia. Chaos control and synchronization of the Newton-Leipnik chaotic system. Chaos, Solitons and Fractals 35 (2008) 814-824.

[21] Yue Wu, Xiaobing Zhou, Jia Chen, Bei Hui. Chaos synchronization of a new 3D chaotic system. Chaos, Solitons and Fractals 42 (2009) 1812-1819.

[22] Her-Terng Yau, Cheng-Shion Shieh. Chaos synchronization using fuzzy logic controller. Nonlinear Analysis: Real World Applications 9 (2008) 1800 - 1810.

[23] Her-Terng Yau, Jun-Juh Yan. Chaos synchronization of different chaotic systems subjected to input nonlinearity. Applied Mathematics and Computation 197 (2008) 775-788.

[24] Mei Sun, Lixin Tian, Changyan Zeng. The energy resources system with parametric perturbations and its hyperchaos control. Nonlinear Analysis: Real World Applications 10 (2009) 2620-2626.

[25] Song Zheng, Gaogao Dong, Qinsheng Bi. A new hyperchaotic system and its synchronization. Applied Mathematics and Computation 215 (2010) 3192-3200.

[26] Sundarapandian Vaidyanathan, HYBRID SYNCHRONIZATION OF HYPERCHAOTIC LIU SYSTEMS VIA SLIDING MODE CONTROL, International Journal of Chaos, Control, Modelling and Simulation (IJCCMS) Vol.1, No.1, September 2012. 
International Journal of Chaos, Control, Modelling and Simulation (IJCCMS) Vol.2, No.1, March 2013

[27] Congxu Zhu. Control and synchronize a novel hyperchaotic system. Applied Mathematics and Computation 216 (2010) 276-284.

[28] BERND BLASIUS and LEWI STONE. CHAOS AND PHASE SYNCHRONIZATION IN ECOLOGICAL SYSTEMS. International Journal of Bifurcation and Chaos, Vol. 10, No. 10 (2000) 2361-2380.

[29] SAEED TAHERION and YING-CHENG LAI. EXPERIMENTAL OBSERVATION OF LAG SYNCHRONIZATION IN COUPLED CHAOTIC SYSTEMS. International Journal of Bifurcation and Chaos, Vol. 10, No. 11 (2000) 2587-2594.

[30] Amir Abbas Emadzadeh, Mohammad Haeri. Anti-Synchronization of two Different Chaotic Systems via Active Control. World Academy of Science, Engineering and Technology 62005.

[31] M. Mossa Al-sawalha, M.S.M. Noorani. Anti-synchronization Between Two Diferent Hyperchaotic Systems. Journal of Uncertain Systems Vol.3, No.3, pp.192-200, 2009.

[32] M. Mossa Al-sawalha and Awni Fayez Al-Dababseh. Nonlinear Anti-Synchronization of Two Hyperchaotic Systems. Applied Mathematical Sciences, Vol.5, 2011, no.38, 1849-1856. 